motion. Such a movement of the background is now one of the well-established facts of astronomy.

A superficial study of the nearer stars suggests that they are moving at random, with differing speeds and in different directions. But a careful statistical investigation reveals law and order in the motions, not of individual stars but of statistical groups. The motion is best described by the statement that each group of stars (sufficient in number to justify statistical treatment, but also comprised within a sufficiently small volume of space) is describing an orbit about a centre. This centre is the same for all groups, and coincides exactly with the centre of the galaxy, as determined by Shapley from the arrangement of the globular clusters. Those groups which are farthest from the centre move most slowly, just as, in the solar system, those planets which are farthest from the sun move most slowly. The reason is, of course, that each star describes an orbit under the gravitational force of the rest of the stars, just as each planet describes an orbit under the gravitational force of the rest of the solar system.

Detailed statistical study of the stars near the sun shows that, on the average, the orbital speed of a star falls off by $1 \mathrm{~km}$. a second for every 200 lightyears increase of distance from the centre of the galaxy. This single datum, which is quite well determined, shows that the sun must take about 250 million years to perform its journey round the centre of the galaxy. Thus it must have completed some ten or a dozen orbits since the earth was born. If the sun is at a distance of 40,000 light-years from the centre of the galaxy, then it must describe its orbit at a speed of about $300 \mathrm{~km}$. a second, a conclusion which agrees well enough with independent estimates made by spectroscopic measurements of the speed of the sun relative to the external galaxies and remote globular clusters.

The various data which have just been mentioned provide the means for weighing the mass which keeps the sun in its orbit, and so the galaxy as a whole. Estimates vary from 110,000 million to 180,000 million times the mass of the sun, so that it seems safe to say that the galaxy contains hundreds of thousands of millions of stars, although the majority are rendered invisible by the thick layer of fog which lies between them and us. It used to be thought that our galaxy was more massive than the others we see in the sky, but this no longer appears to be the case. We are familiar with groups of stars which are held together by their mutual gravitational attractionsthe globular clusters provide an instance. There are also clusters of nebulæ which are held together in the same way. It is possible to determine the speeds of motion of the individual nebulæ of a cluster, and so deduce the gravitational forces needed to hold the cluster together. In this way, the average galaxy is found to have a mass of the order of from 100,000 million to 200,000 million suns. Thus there can be no doubt that the external galaxies are at least comparable with our own galaxy in mass.

Finally, it used to be thought that the external galaxies are substantially smaller than our own in size, but it has recently emerged that this too is fallacious. We only see a small part of a galaxy when we study its apparent size on a photographic plate; there is a much larger part beyond, which can only be detected by delicate photometric measurements. When we take this into account, the galaxies still show considerable differences in size, but the majority of the larger prove to be comparable with our own. On the whole, then, our galaxy is simply one of many similar galaxies. Probably about four million such can be seen photographically in the great Mt. Wilson telescope. If we allot 100,000 million stars to each, this makes a total of about $4 \times 10^{17}$ stars-a large number, although still small in comparison with the number of molecules in a cubic centimetre of ordinary air.

\section{REGIONAL PLANT ECOLOGY IN THE UNITED STATES}

\author{
By Dr. V. J. CHAPMAN \\ Botany School, Cambridge
}

$\mathrm{T}$

HE last four years has seen the publication, by J. H. Davis, of three important papers on the vegetation of Southern Florida*. These three valuable papers yield a clear picture of the vegetation and its interrelations with soil types, elimate and physio. graphy. The area is especially interesting because climax vegetation is normally related to the climate, but Davis establishes a good case in this area for relating it primarily to the physiography. If the author continues these studies - as one sincerely hopes he will-the vegetation of Southern Florida will be known and understood in very considerable detail. This will be no mean feat for an area of such size. The majority of ecological studies usually refer to relatively restricted areas, and it is refreshing to find a study that embraces such a large region. A broad survey with accompanying detailed studies opens up major problems that would not be so evident in a study of a small area.

In the first paper, on the mangrove vegetation, Davis points out that they are primarily edaphic forests, a conclusion with which I agree, though I would add that there is also a physiographic element involved. Tropical forest of the 'ham. mock' type is regarded as the climax vegetation, though in the third paper it is also, albeit incorrectly, implied that mangroves represent a climax type. There is no evidence of a transition to brackish or freshwater marsh such as may be found in Jamaica. Davis studied the environmental factors in some detail and he shows that the mangrove species possess a wide tolerance of salinity and grow on at least four types of soil, one of which is a marine peat. I have arrived at similar conclusions from work in Jamaica. Davis relates the mangrove zonation to the height of the surface water, but here one feels that more evidence would be desirable. Some exceedingly interesting information is provided about dispersal. 10,000 Rhizophora seedlings are estimated to float towards the Tortugas every year; this represents only a proportion of the total crop because about 50 per cent of the seedlings from a tree remain embedded in the mud beneath it. The rate of sur. vival at the end of one year is 50 per cent for Rhizophora, 30 per cent for Avicennia and 20 per cent for Laguncularia.

One of the important features of these three papers is the great use made of aerial photography in the study of the communities and the preparation of the

* Davis, J. H., "The Ecology and Geologic Rôles of Mangroves in Florida", Carn. Inst. Wash. Pub., 517 (1940); "The Ecology of the Vegetation and Topography of the Sand Keys of Florida", Carn Inst. Wash. Pub. $524(1942)$; The Natural Features of Southern
Florida", State of Florida Dept. of Conservation, Bull. 25 (1943). 
vegetation maps. At the present time vast quantities of air photographs of Europe, Africa and elsewhere must be accumulating, and it is to be hoped that they will become available to ecologists, who will then be in a position to study vegetation on a scale comparable to that of Davis.

The second paper, on the sand keys of Florida, is of particular interest because it adds a new set of vegetation maps to the series started by Millspaugh in 1907 and continued by Bowman in 1917. Changes in the vegetation of these keys over a period of thirty-seven years can therefore be studied. The Marquesas, like Jamaica, have few or no living corals, whereas living corals abound in the Tortugas. This is a peculiar feature of the Caribbean and one which greatly needs investigation. Davis concludes that marine currents are the main agents in the formation of these keys; but it is elear also that hurricanes have had a profound influence in the past upon their structure and also their vegetation. Two types of vegetation are recognized, the sea-strand and the mangrove swamp. The use of habitat is employed in the ecological nomenclature. This has been discouraged by most ecologists working in temperate climates, but tropical vegetation, and especially that of maritime regions, appears to have problems of its own. It may eventually prove desirable in such cases to employ a habitat nomenclature. I encountered a similar difficulty in Jamaica. The number of species recorded by Davis is not large; but this is typical of small islands of this type. The climates of the island groups vary sufficiently for the biological life-form spectra to show significant differences. The Schimperian view of 'physiological dryness' is adopted for the mangrove habitat; but the evidence available to-day scarcely supports such an interpretation.

The third paper covers the whole of Southern Florida, and all the various features are considered with a view to future regional utilization and planning. Aeroplanes, cars, special tractors and 'airboats' enabled the author to visit many areas that were previously almost inaccessible. Much of the region is, of course, covered by the famous Everglades. 'Glades' are grasslands flanked by forests, the 'ever' being added to signify that they remain green throughout the year. Much of the Everglades is dominated by the saw-grass, Mariscus jamaicensis, but there are also prairies, sloughs and 'hammocks'. These swamps originated as a result of regular seasonal flooding. The 'dismal' swamps with pine and eypress do not form part of the glades. The whole area can be regarded as a huge alkaline peat-bog, in many ways comparable to what the British Fens must have looked like in the past. Vast deposits of peat are here being formed under subtropical conditions. Unfortunately the natural water conditions have been upset and this is bringing about changes in the organic soils and the vegetation. The area is becoming drier and saw-grass is being replaced by bushes (= carr stage of Wicken Fen). The annual transpiration and evaporation from the saw-grass area is often more than the annual rainfall, and hence the water relations are closely bound up with those

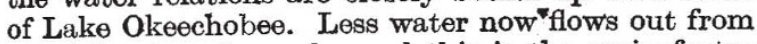
this lake than formerly, and this is the main factor responsible for the present changes. Piles of marine shells found inland indicate past ease of travel by innumerable waterways many of which have now silted up. There is evidently scope here for a combination of archæological and botanical research in a manner similar to that so successfully carried out by Godwin and his co-workers in Great Britain.

Ten physiographic regions are recognized for Southern Florida and at least three old shore lines. The sands left by these seas form the main shallow sand soils in which little or no profile has developed. Beneath the Everglades there is an impervious marl layer, and Davis believes that these swamps would never have arisen were it not for this marl layer which effectively impedes drainage. If this is true, then the Everglades are related neither to the present climate nor to existing physiography but to past geological changes. Although twenty soil types are recognized, peats and marls are the outstanding features of this region. The soils are generally shallow and they have been much" reduced by fires, which form an important determining factor of the environment. A detailed correlation is made between the different vegetation communities, individual species. and the soil types.

A study of the water relations showed that the ratio, time water-logged to time dry, is the most important factor. Because of the low relief a few inches change in elevation makes a profound differ. ence in the.drainage relations.

Nine main types of vegetation are recognized and fifteen types are represented on the vegetation map. There is a very high proportion of woody plants, and Davis concludes that a.t least one half of the area is economically unprofitable and best left in the wild state. The characteristic 'hammock' forests represent the climax vegetation in Southern Florida. These possess a great diversity of plants, many of the species being tropical. If the fire danger were controlled these forests would be larger, while even at present their arrangement provides an indication of the drainage relations. Davis also considers that the cypresses (Taxodium) will not invade an area where water is continually standing on the surface because the seedlings require atmospheric oxygen.

These three papers contain many more valuable observations, but sufficient has been said to indicate their scope and importance.

\section{BIOLOGY IN WAR-TIME CHINA*}

\author{
BY DR. PEI-SUNG TANG \\ Director of the Tsing Hua University Physiological
Laboratory
}

$T$ HIS article will be of the nature of a report on the movements of biological institutions during the War and the activities of biologists associated with those institutions. No attempt will be made to survey the entire field of biology in war-time China, or to evaluate the work at present being done by Chinese biologists.

During the decade immediately preceding the War, there were several centres of biological investigation in China from which came a steady output of research. There were, for example, the physiologists at the Peiping Union Medical College, gathered round Robert K. S. Limt, who virtually founded the science of physiology in China; the biochemists, whom $H$. Wu brought together in the same College; the Fan

* Condensed version.

$\dagger$ The Romanized names of the biologists mentioned in this article are those used by the authors in their publications. 